\title{
Effect of Calibration Error on Reconstruction Accuracy of Stereovision System
}

\author{
Jianhua Wang ${ }^{*}$, Zhao Yang and Yuping Wu
}

Marine Technology \& Control Engineering Key Laboratory, Shanghai Maritime University, Shanghai, 201306, P. R. of China

\begin{abstract}
This paper investigates the relation between the calibration error and reconstruction accuracy of stereovision system through simulation experiment. According to the model of a typical non-parallel stereovision system, an array of points in the common view field of the two cameras is projected onto the image plane of the left and right camera respectively, forming the left image and right image. After changing the calibration parameter slightly around its ground truth, the array of points is reconstructed. By comparing the reconstruction result of the points in the array with their original position, the major factors affecting reconstruction accuracy are summarized, which include the abscissa of the principal point, the component of the rotation vector along the ordinate axis, the first order radial distortion coefficient, the component of the translation vector along the abscissa axis, and the abscissa of the principal length. Especially, the abscissa of the principal point and the component of the rotation vector along the ordinate axis have prominent effect on reconstruction accuracy. In the process of calibration, the error of the major factors must be controlled strictly, so that the accuracy requirement of the stereovision system can be satisfied.
\end{abstract}

Keywords: Stereovision, Reconstruction Accuracy, Calibration Error, Intrinsic Parameter, Extrinsic Parameter.

\section{INTRODUCTION}

Stereovision is an effective means of 3D measurement, scene understanding and obstacle avoidance for autonomous robotic vehicles. It has been one of the most attractive topics in computer vision and robotics $[1,2]$. Main stages in stereovision include calibration, correspondence and reconstruction. Accuracy analysis in stereovision is discussed in [3-7], wherein references $[4,5]$ are focused on correspondence while $[6,7]$ pay attention to reconstruction. In practical application, we often encounter the following problem. In what accuracy does the stereovision system need calibrating so that the result of reconstruction can satisfy the required accuracy? Motivated by the problem, this paper investigates the relation between the calibration error and reconstruction accuracy.

Theoretically, analysis of the relation between the calibration error and reconstruction accuracy is much complicated, because reconstruction is involved in transformation of matrix including more than 16 calibration parameters, and iterative process is usually necessary. In an applicable stereovision system, the calibration parameter changes only in a small range around its ground truth when error is considered. Consequently, simulation experiment around ground truth is an effective and simple method to explore the relation between the calibration error and reconstruction accuracy of a stereovision system.

*Address correspondence to this author at the Marine Technology \& Control Engineering Key Laboratory, Shanghai Maritime University, Shanghai, 201306, P. R. of China; Tel: 86-21-38284649; Fax: 86-21-38284643;

E-mail: jhwang@shmtu.edu.cn
Suppose the stereovision system consists of two cameras with known calibration parameters, and an array of points with known 3D coordinates is located in the common view field of the two cameras. At first, project the array of points onto the image plane of the two cameras respectively according to the projecting model with given calibration parameters (ground truth), forming the left image and the right image. Then, change the calibration parameter slightly around its ground truth, and reconstruct the array of points using the changed parameter. Through comparison of the reconstruction results from the changed parameters and from the ground truth, the major factors affecting reconstruction accuracy can be found.

\section{SIMULATION MODEL}

The simulation model includes projection and reconstruction. They are addressed below respectively.

\subsection{Projecting Model}

Fig. (1) shows a typical non-parallel stereovision system. Suppose the intrinsic parameters of its left camera and right camera are expressed by matrix $\mathbf{A}_{l}$ and $\mathbf{A}_{r}$ respectively, as shown in Eq. (1), where $i=l$ or $r . \mathbf{P}_{0 l}\left(u_{o l}, v_{o l}\right)$ and $\mathbf{P}_{0 r}\left(u_{0 r}, v_{0 r}\right)$ are the principal points of the left camera and right camera respectively, with $\alpha_{l}, \beta_{l}$ and $\alpha_{r}, \beta_{r}$ being their principal lengths.

$$
\mathbf{A}_{i}=\left[\begin{array}{ccc}
\alpha_{i} & 0 & u_{0 i} \\
0 & \beta_{i} & v_{0 i} \\
0 & 0 & 1
\end{array}\right]
$$




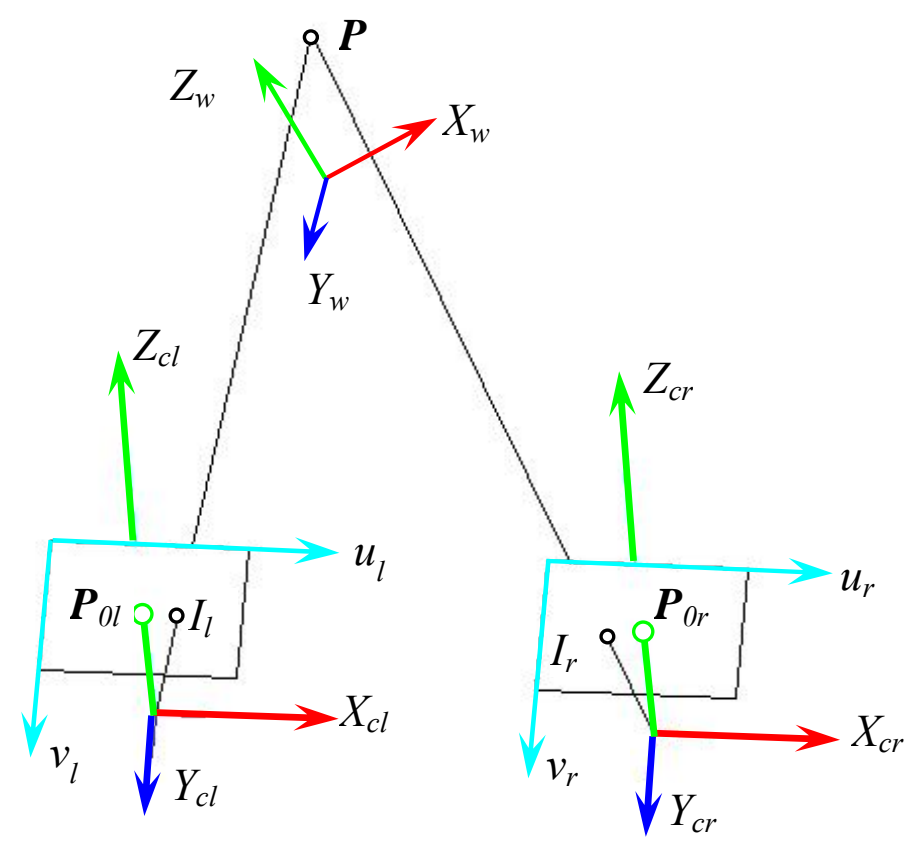

Fig. (1). Stereovision geometry.

The rotation matrix of the right camera with respect to the left camera is $\mathbf{R}$.

$\mathbf{R}=\left[\begin{array}{lll}r_{11} & r_{12} & r_{13} \\ r_{21} & r_{22} & r_{23} \\ r_{31} & r_{32} & r_{33}\end{array}\right]$

According to Rodrigues formula [8], the rotation matrix can be expressed by the rotation vector $\mathbf{n}$.

$\mathbf{n}=\left[\begin{array}{lll}n_{1} & n_{2} & n_{3}\end{array}\right]^{T}$

The translation vector of the right camera with respect to the left camera is $\mathbf{T}$.

$$
\mathbf{T}=\left[\begin{array}{lll}
t_{1} & t_{2} & t_{3}
\end{array}\right]^{T}
$$

Suppose the point $\mathbf{P}$ in the world frame $X_{w} Y_{w} Z_{w}$ is in the common view field of the left camera and the right camera, and its coordinates in the left camera frame are $\mathbf{P}_{c l}\left(\mathrm{X}_{c l}, \mathrm{Y}_{c l}\right.$, $\left.\mathrm{Z}_{c l}\right)$. Then its coordinates in the right camera frame $\mathbf{P}_{c r}\left(\mathrm{X}_{c r}\right.$, $\left.\mathrm{Y}_{c r}, \mathrm{Z}_{c r}\right)$ can be found by the following relation.

$$
\left[\begin{array}{c}
X_{c r} \\
Y_{c r} \\
Z_{c r}
\end{array}\right]=\mathbf{R}\left[\begin{array}{c}
X_{c l} \\
Y_{c l} \\
Z_{c l}
\end{array}\right]+\mathbf{T}
$$

Suppose $\mathbf{I}_{l}\left(u_{l}, v_{l}\right)$ and $\mathbf{I}_{r}\left(u_{r}, v_{r}\right)$ are a pair of conjugate points on the left image plane and the right image plane from the same point $\mathbf{P}$ in the common field of view, then they can be obtained from the following formula, where $i=l$ or $r$.

$$
Z_{c i}\left[\begin{array}{l}
u_{i} \\
v_{i} \\
1
\end{array}\right]=\left[\begin{array}{ccc}
\alpha_{i} & 0 & u_{0 i} \\
0 & \beta_{i} & v_{0 i} \\
0 & 0 & 1
\end{array}\right]\left[\begin{array}{l}
X_{c i} \\
Y_{c i} \\
Z_{c i}
\end{array}\right]
$$

If we consider only the first order radial distortion of the lenses in the cameras, the observed image points $\mathbf{I}_{i d}\left(u_{i d}, v_{i d}\right)$ can be obtained through the following distortion model.

$\left[\begin{array}{l}u_{i d} \\ v_{i d}\end{array}\right]=\left[\begin{array}{l}u_{i} \\ v_{i}\end{array}\right]+k_{1 i}\left[\left(u_{i}-u_{0 i}\right)^{2}+\left(v_{i}-v_{0 i}\right)^{2}\right]\left[\begin{array}{l}u_{i} \\ v_{i}\end{array}\right]$

\subsection{Reconstructing Model}

Stereovision aims to recover 3D coordinates $\mathbf{P}_{c l}$ or $\mathbf{P}_{c r}$ of a point in the common field of view from its $2 \mathrm{D}$ image coordinates $\mathbf{I}_{l}\left(u_{l}, v_{l}\right)$ and $\mathbf{I}_{r}\left(u_{r}, v_{r}\right)$. Before reconstruction computation, the lens distortion needs correcting, and this can be done by the following equation.

$\left[\begin{array}{c}u_{i} \\ v_{i}\end{array}\right]=\frac{1}{1+k_{1 i}\left[\left(u_{i}-u_{0 i}\right)^{2}+\left(v_{i}-v_{0 i}\right)^{2}\right]}\left[\begin{array}{l}u_{i d} \\ v_{i d}\end{array}\right]$

For the conventional configuration of stereovision system, which includes a pair of cameras with same intrinsic parameters and parallel camera coordinate systems only separated by a horizontal distance, it is well known that the coordinates of a scene point can be easily obtained from its disparities. In practice, however, most stereovision systems are nonparallel configuration. The typical approach involved in reconstruction from nonparallel stereovision so far is through rotating the left and right camera coordinate systems to make them satisfy the conventional parallel stereovision geometry, and then is employed the standard algorithm [7].

Suppose the unit vector along the $\mathrm{X}_{c l}$ direction in the left camera frame is $\mathbf{x}$, then the unit rotation vector $\mathbf{q}$ of the left camera can be found by Eq.(9), and the rotation angle can be computed by Eq.(10). According to Rodrigues formula [8], the rotation matrix of the left camera can be obtained by Eq. (11). 


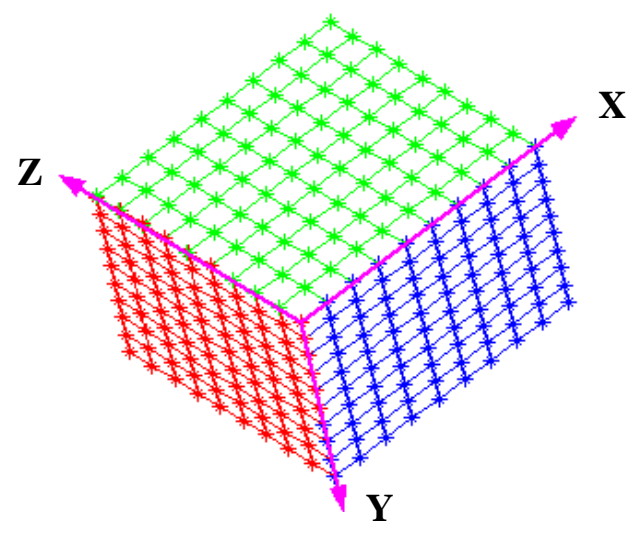

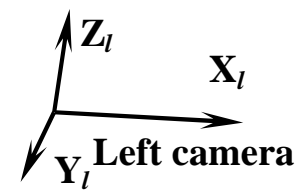

Fig. (2). Configure of the stereovision.

$\mathbf{q}=\left[\begin{array}{lll}q_{1} & q_{2} & q_{3}\end{array}\right]^{T}=\frac{\mathbf{x} \times \mathbf{T}}{\|\mathbf{x} \times \mathbf{T}\|}$

$\theta=\arccos \frac{\mathbf{x} \bullet \mathbf{T}}{\|\mathbf{x} \bullet \mathbf{T}\|}$

$\mathbf{R}_{c l}=\mathbf{E}+\mathbf{S}_{n} \sin \theta+\mathbf{S}_{n}^{2}(1-\cos \theta)$

where $\mathbf{E}$ is the identity matrix, and

$\mathbf{S}_{n}=\left[\begin{array}{ccc}0 & -q_{3} & q_{2} \\ -q_{3} & 0 & q_{1} \\ q_{2} & q_{1} & 0\end{array}\right]$

According to the Eq. (5), the rotation matrix of the right camera can be obtained by Eq.(13).

$\mathbf{R}_{c r}=\mathbf{R}_{c l} \mathbf{R}$

For the image points $\mathbf{I}_{l}\left(u_{l}, v_{l}\right)$ and $\mathbf{I}_{r}\left(u_{r}, v_{r}\right)$, their normalized coordinates $\boldsymbol{N}_{l}$ or $\boldsymbol{N}_{r}$ can be derived by Eq.(14).

$\mathbf{N}_{i}=\left[\begin{array}{ccc}\frac{u_{i}-u_{0 i}}{\alpha_{i}} & \frac{v_{i}-v_{0 i}}{\beta_{i}} & 1\end{array}\right]^{T}$

By applying rotation $\mathbf{R}_{c l}$ and $\mathbf{R}_{c r}$ to $\mathbf{N}_{l}$ and $\mathbf{N}_{r}$ respectively, they are transformed to $\mathbf{N}_{l T}\left(u_{l T}, v_{l T}\right)$ and $\mathbf{N}_{r T}\left(u_{r T}, v_{r T}\right)$.

$\mathbf{N}_{i T}=\mathbf{R}_{c i} \mathbf{N}_{i}=\left[\begin{array}{lll}n_{i T 1} & n_{i T 2} & n_{i T 3}\end{array}\right]^{T}$

After rotation, the left and right cameras consist of a parallel stereovision configuration with baseline $B=\|\mathbf{T}\|$, and the image points $\mathbf{I}_{l}\left(u_{l}, v_{l}\right)$ and $\mathbf{I}_{r}\left(u_{r}, v_{r}\right)$ are transformed to $\mathbf{I}_{l T}\left(u_{l T}, v_{l T}\right)$ and $\mathbf{I}_{r T}\left(u_{r T}, v_{r T}\right)$ respectively.

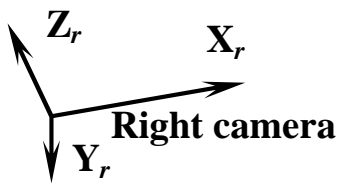

By employing the standard algorithm of parallel stereovision, 3D coordinates of point $\mathbf{P}$ in the left camera frame can be obtained by Eq.(17)

$$
\mathbf{P}_{c l T}=\frac{B}{u_{l T}-u_{r T}}\left[\begin{array}{ccc}
u_{l T}-u_{l 0} \frac{\alpha_{l}}{\beta_{l}}\left(v_{l T}-v_{l 0}\right) & \alpha_{l}
\end{array}\right]^{T}
$$

Finally, transform the $3 \mathrm{D}$ coordinates $\mathbf{P}_{c l T}$ from the rotated left frame to the original left frame, and the reconstructed 3D point $\mathbf{P}_{c l r}$ is obtained.

$$
\mathbf{P}_{c l r}=\mathbf{R}_{c l}^{T} \mathbf{P}_{c l T}
$$

If the intrinsic parameters of the two cameras, as well as the extrinsic parameters expressing relative position and position of the left camera with respect to the right camera, are the same in reconstruction as in projection, the reconstructed point $\mathbf{P}_{c l r}$ is coincident with the original one $\mathbf{P}_{c l}$, i.e. $\mathbf{P}_{c l r}=\mathbf{P}_{c l}$. If one of the intrinsic parameters or extrinsic parameters changes, the reconstructed point $\mathbf{P}_{c l r}$ will differ from the original one $\mathbf{P}_{c l}$, and the error $\delta \mathbf{P}_{c l}$ occurs.

$\delta \mathbf{P}_{c l}=\mathbf{P}_{c l r}-\mathbf{P}_{c l}$

By investigating the reconstruction error $\delta \mathbf{P}_{c l}$, the effect of calibration error on accuracy of reconstruction can be discussed.

\section{SIMULATION EXPERIMENT}

In the simulation experiment, the stereovision system is configured as shown in Fig. (2). Suppose the intrinsic parameter matrix of the left camera is $\mathbf{A}_{l}=\left[\begin{array}{ll}5400 & 0 \\ 500 ; 0 & 5400\end{array}\right.$ 400; 00 1], and $\mathbf{A}_{r}=\mathbf{A}_{l}$; the rotation vector and translation 


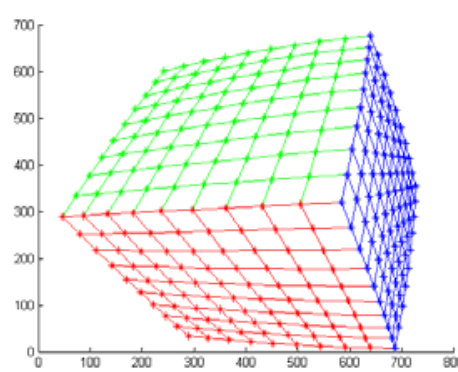

(a)

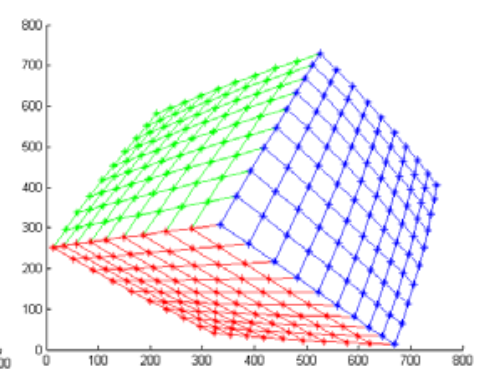

(b)

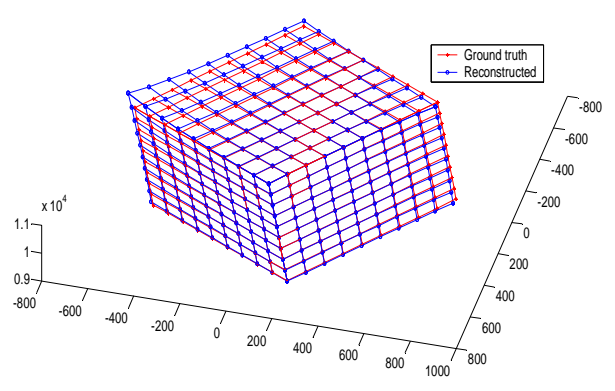

(c)

Fig. (3). Projected images and reconstructed array.

(a) left image; (b) right image; (c) reconstructed array.

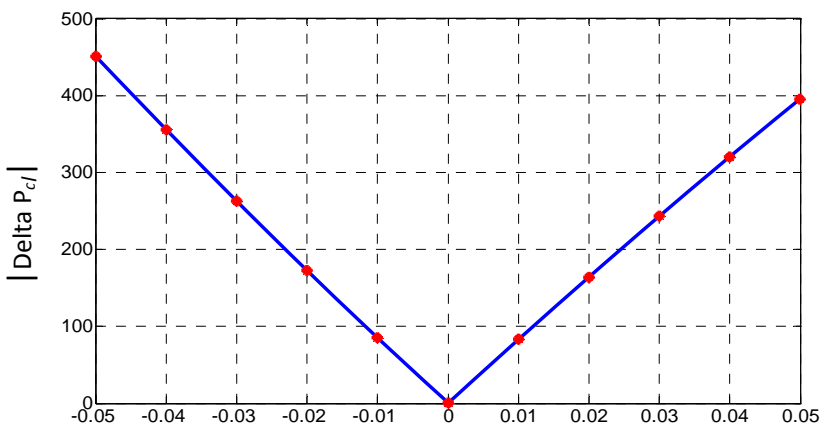

(a)

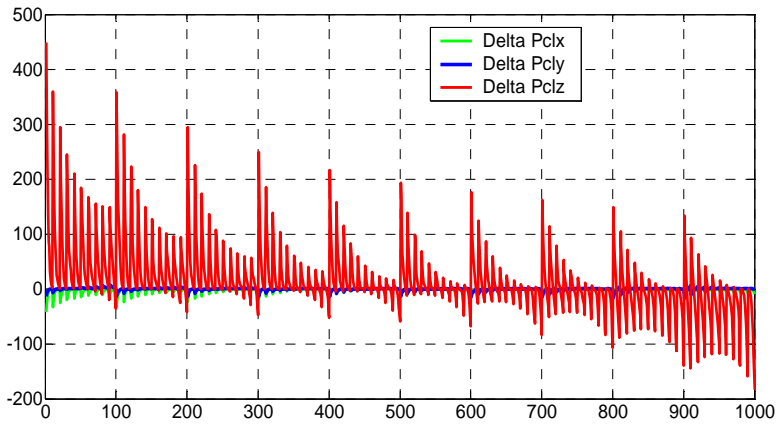

(b)

Fig. (4). Effect of the first order radial distortion $\mathrm{k}_{1 l}$

(a) The maximum error versus $\mathrm{k}_{11}$; (b) Components of error versus ordinal of point

vector of the right camera with respect to the left camera are $\mathbf{n}=[0.00996 ;-0.02711 ; 0.01749]$ and $\mathbf{T}=[150 ; 5 ; 30]$ respectively; the first order radial distortion coefficient of the lens in the left camera is $\mathrm{k}_{1 l}=-5.3 \times 10^{-7}$, and $\mathrm{k}_{1 r}=\mathrm{k}_{1 l}$. The above parameters are from a stereovision system used in an autonomous surface vehicle.

A three dimensional array of points including $10 \times 10 \times 10=1000$ points is supposed to be in the common field of view. The distance between two adjacent points in the array is $100 \mathrm{~mm}$. The rotation matrix and translation vector of the array frame $\mathrm{XYZ}$ with respect to the left camera frame $\mathrm{X}_{l} \mathrm{Y}_{l} \mathrm{Z}_{l}$ are $\mathbf{R a}=\left[\begin{array}{llll}0.7220 & 0.2780 & 0.6336 ; 0.2780 & 0.7220\end{array}\right.$ -0.6336; $-0.63360 .63360 .4440]$ and $\mathbf{T a}=[-280 ; 360 ; 10000]$. Each point in the array can be expressed in the left camera frame as $\mathbf{P}_{c l}$, which is referred to as original position.

The array of points is projected onto the left and right camera respectively according to the projecting model from Eq.(1) to Eq.(7) with above parameters, forming the left image and the right image as shown in Fig. (3a) and Fig. (3b). From the left image and the right image, the array of point is reconstructed according to the algorithm from Eq.(8) to Eq.(18) with calibration parameters changed slightly around the ground truth. An example of reconstructed results is shown in Fig. 3(c), where the red stars denote the original position of the points in the array, and the blue circles denote the reconstructed position. It can be seen that when one of the calibration parameters is changed slightly around the ground truth, the reconstructed position of the points differs from the original position. Then, how and in what a degree does each of the calibration parameters affect the reconstruct accuracy? It will be demonstrated in the following.

\subsection{Effect of Lens Distortion}

Change the first order radial distortion coefficient of the left lens $\mathrm{k}_{1 l}$ in the range of $\pm 5 \%$ around its ground truth with step size of $1 \%$, and reconstruct the array of points at each step. The maximum position error $\left|\delta \mathbf{P}_{c l}\right|$ of the points in the array at each step is shown in Fig. (4a). It can be seen that the maximum position error is about $450 \mathrm{~mm}$ when the $\mathrm{k}_{1 l}$ is changed by $-5 \%$; the relation between increment of $\mathrm{k}_{1 l}$ and the position error is nearly linear.

When the increment of $\mathrm{k}_{1 l}$ is $5 \%$, the position error of each point in the array is shown in Fig. (4b). The points are numbered from left to right, from top to bottom, and from near to far with respect to the left camera. It can be seen that the error is small when the point lies near the center of the array. According to the given position of the array, when the point lies near the center of the array, its image is near the center of the image plane, as shown in Fig. (3a) and Fig. (3) (b), where the lens distortion is small, and so the reconstruction error. In addition, the depth component $\delta \mathrm{P}_{c l z}$ is prominent compared with the other components of the error vector.

\subsection{Effect of the Principal Point}

Change the principal point coordinate $u_{0 l}$ or $v_{0 l}$ of the left camera in the range of \pm 5 pixels around its ground truth with 


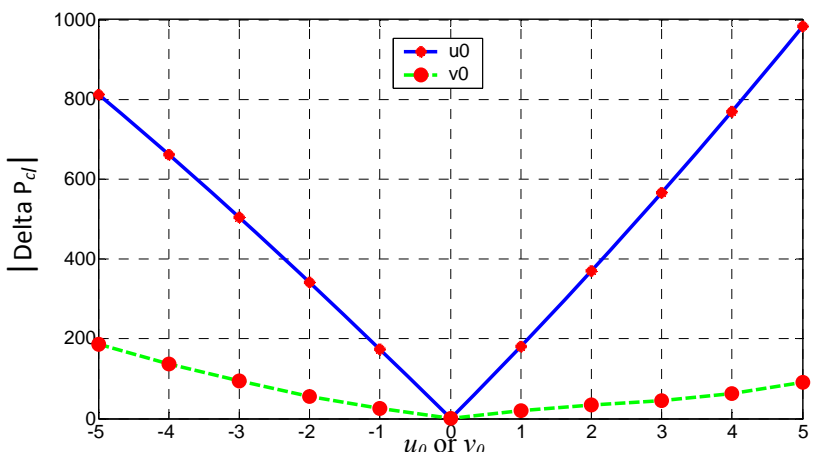

(a)

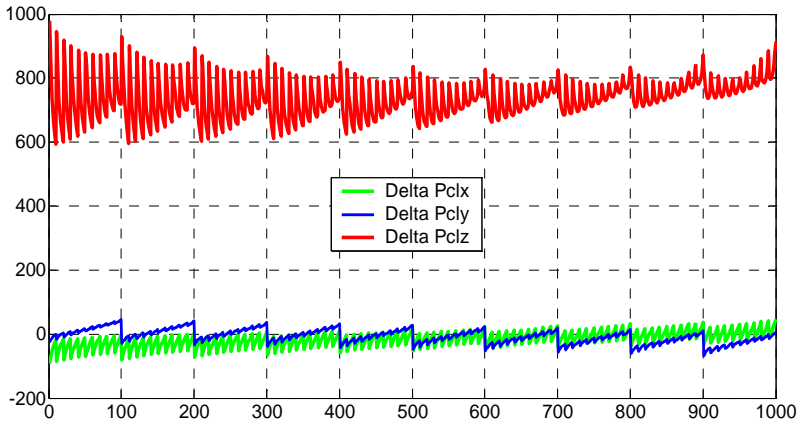

(b)

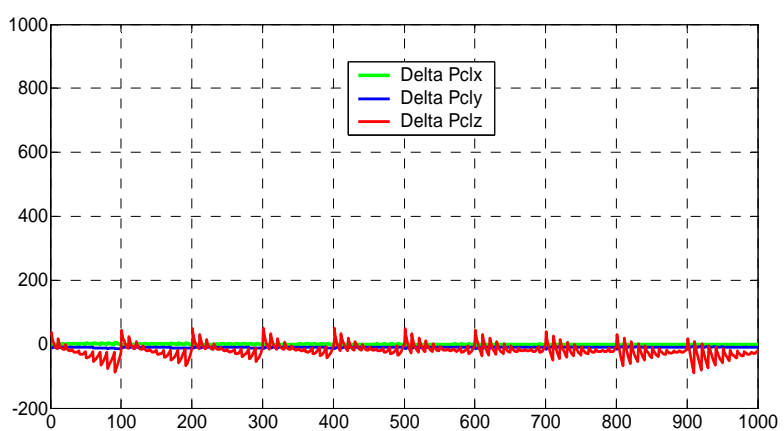

(c)

Fig. (5). Effect of principle point

(a) Reconstruction error versus deviation of the principle point (b) Components of error versus point ordinal when $u_{0}$ changed

(c) Components of error versus point ordinal when $v_{0}$ changed

step size of 1 pixel and reconstruct the array of points at each step. The maximum position error $\left|\delta \mathbf{P}_{c l}\right|$ of the points in the array at each step is shown in Fig. 5(a). It can be seen that the maximum error is about $1000 \mathrm{~mm}$ when the $u_{0 l}$ changes by 5 pixels, while the maximum error is only about $200 \mathrm{~mm}$ when the $v_{0 l}$ changes by 5 pixels. Obviously, abscissa error of the principal point is one of the main factors affecting reconstruction accuracy.

When $u_{0 l}$ is changed by 5 pixels, the error of each point in the array is shown in Fig. 5(b). It can be seen that the depth component $\delta \mathrm{P}_{c l z}$ is prominent compared with the other components. Unlike the effect of lens distortion, the depth component $\delta \mathrm{P}_{c l z}$ of the error vector caused by $u_{0 l}$ has an average far from zero.

When $v_{0 l}$ is changed by 5 pixels, the error of each point in the array is shown in Fig. 5(c). It can be seen that the depth

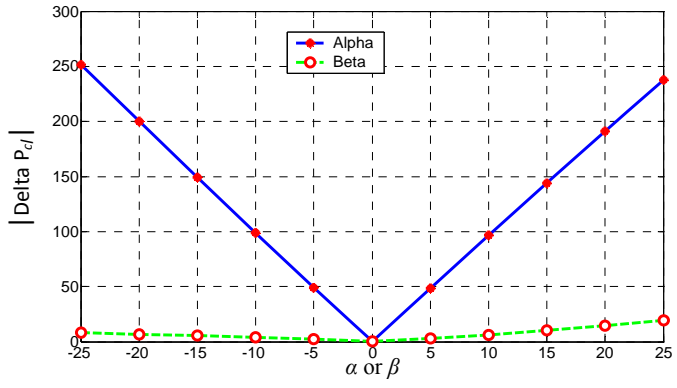

(a)

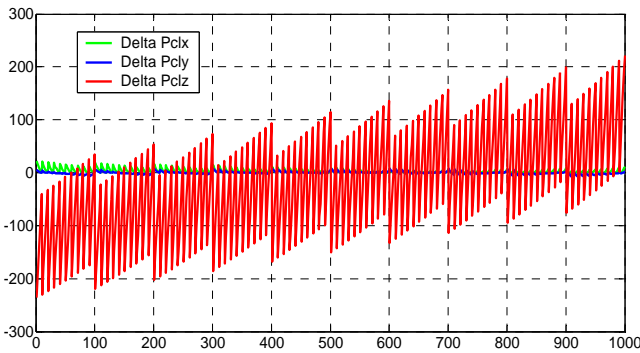

(b)

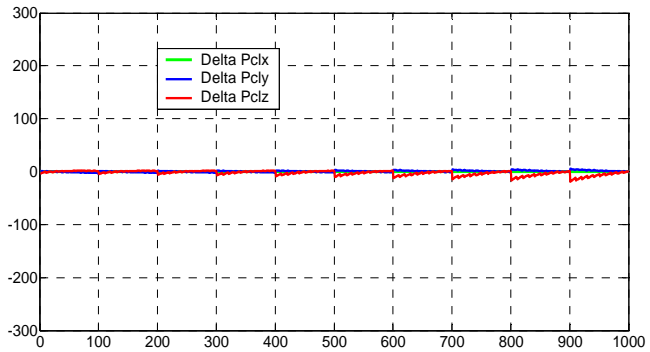

(c)

Fig. (6). Effect of principle length

(a) Reconstruction error versus deviation of the principle length

(b) Components of error versus point ordinal when $\alpha$ changes

(c) Components of error versus point ordinal when $\beta$ changes

component $\delta \mathrm{P}_{c l z}$ has bigger change than the other components.

Comparing the effect of $u_{0}$ and $v_{0}$ according to Fig. 5(b) and Fig. 5(c), it is obvious that the reconstruction error caused by $u_{0}$ is about 5 times the one caused by $v_{0}$. Consequently, the abscissa of the principal point is one of the major factors affecting reconstruction accuracy.

\subsection{Effect of the Principal Length}

Change the principal length $\alpha_{l}$ or $\beta_{l}$ of the left camera in the range of \pm 25 pixels around its ground truth with step size of 5 pixels and reconstruct the array of points at each step. The maximum position error $\left|\delta \mathbf{P}_{c l}\right|$ of the points in the array at each step is shown in Fig. 6(a). It can be seen that the maximum error is only about $250 \mathrm{~mm}$ when $\alpha_{l}$ changes by 25 pixels, while the maximum error is much smaller when $\beta_{l}$ changes by 25 pixels.

When $\alpha_{l}$ is changed by 25 pixels, the error of each point in the array is shown in Fig. 6(b). It can be seen that the depth component $\delta \mathrm{P}_{c l z}$ is prominent compared with the other components of the error vector. 


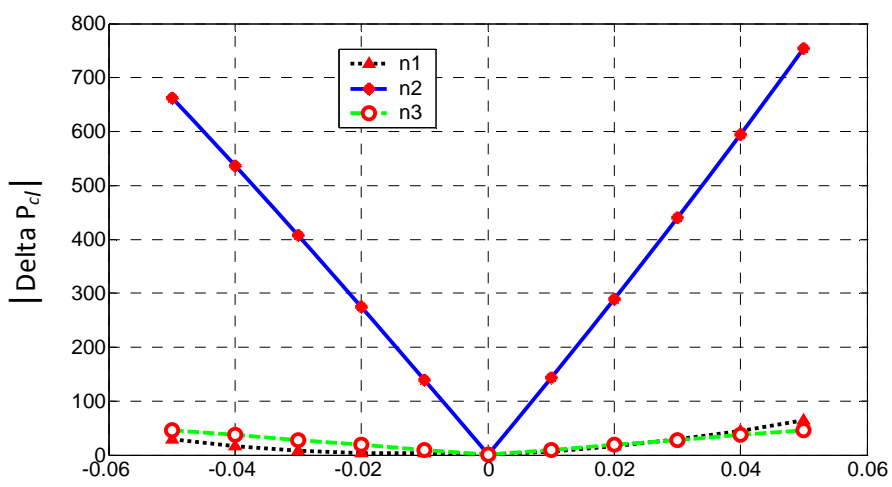

(a)

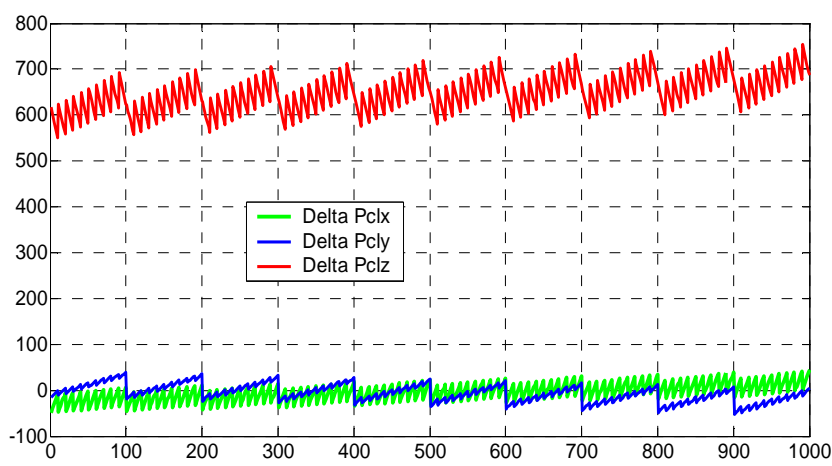

(c)

Fig. (7). Effect of rotation vector

(a) Error versus deviation of the rotattion vector;

(b) Components of error versus point ordinal when $n 1$ changes

(c) Components of error versus point ordinal when $n 2$ changes;

(d) Components of error versus point ordinal when $n 3$ changes

When $\beta_{l}$ is changed by 25 pixels, the error of each point in the array is shown in Fig. (6c). It can be seen that $\beta$ has little effect on the reconstruction error.

In general, calibration error of the principal length is not a main factor affecting reconstruct accuracy.

\subsection{Effect of the Rotation Vector}

Change the components $n_{1}, n_{2}$ and $n_{3}$ of the rotation vector $\mathbf{n}$ in the range of \pm 0.05 degrees around its ground truth with step size of 0.01 degrees and reconstruct the array of points at each step. The maximum position error $\left|\delta \mathbf{P}_{c l}\right|$ of the points in the array at each step is shown in Fig. (7a). It can be seen that the maximum error is about $750 \mathrm{~mm}$ when $n_{2}$ changes by 0.05 degrees, while the maximum error is smaller than $100 \mathrm{~mm}$ when $n_{1}$ or $n_{3}$ changes by 0.05 degrees. Obviously, the reconstruction error caused by $n_{2}$ is about 10 times the one caused by $n_{1}$ or $n_{3}$.

When each component of the rotation vector is changed by 0.05 degrees, the error of each point in the array is shown in Fig. (7b), Fig. (7c) and Fig. (7d). It can be seen that the second component of the rotation vector is another major factor affecting the reconstruction accuracy.

\subsection{Effect of the Translation Vector}

Change the components $t_{1}, t_{2}$ and $t_{3}$ of the translational vector $\mathbf{T}$ in the range of $5 \mathrm{~mm}$ around its ground truth with

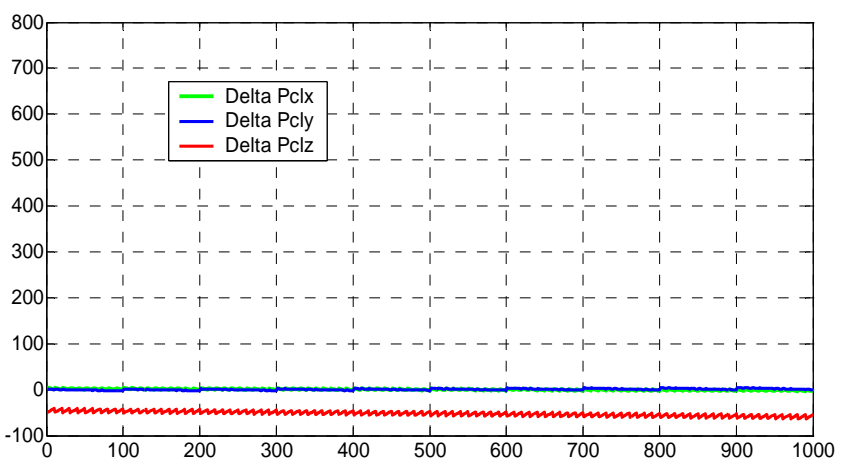

(b)

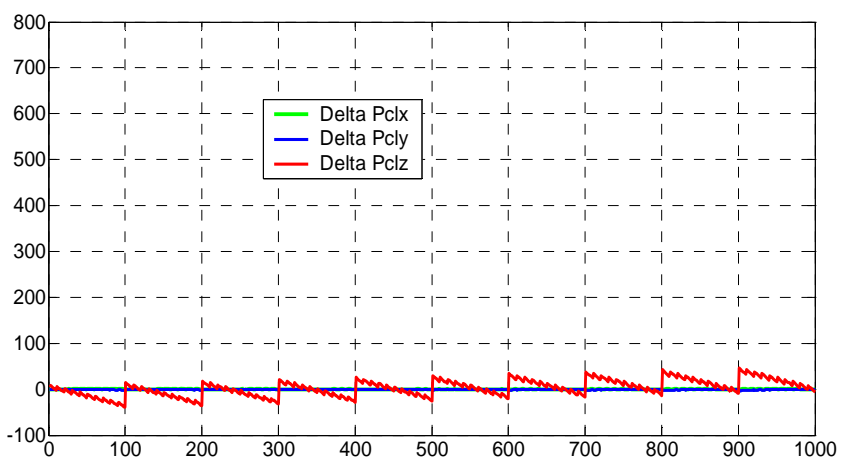

(d)

step size of $1 \mathrm{~mm}$ and reconstruct the array of points at each step. The maximum position error $\left|\delta \mathbf{P}_{c l}\right|$ of the points in the array at each step is shown in Fig. (8a). It can be seen that the maximum error is about $370 \mathrm{~mm}$ when $t_{1}$ changes by 5 $\mathrm{mm}$, while the maximum errors are smaller than $50 \mathrm{~mm}$ when $t_{2}$ or $t_{3}$ changes by $5 \mathrm{~mm}$. Obviously the reconstruction error caused by $t_{1}$ is about 10 times the one caused by $t_{2}$ or $t_{2}$.

When each component of the translation vector is changed by $5 \mathrm{~mm}$, the error of each point in the array is shown in Fig. (8b), Fig. (8c) and Fig. (8d). It can be seen that the first component of the translation vector has great effect on reconstruction accuracy, while the second and the third components of the translation vector have little effect on the reconstruction accuracy.

\subsection{Summary of Experiment Results}

For a typical stereovision system, there are 16 calibration parameters, including intrinsic parameters of the two cameras $\left[u_{0 l} v_{0 l} \alpha_{l} \beta_{l} \mathrm{k}_{1 l}\right],\left[u_{0 \mathrm{r}} v_{0 r} \alpha_{r} \beta_{r} \mathrm{k}_{1 r}\right]$, and extrinsic parameters $\left[n_{1} n_{2} n_{3} t_{1} t_{2} t_{3}\right]$. However, the effect of these parameters on reconstruction accuracy is different. According to the effect degree, calibration parameters can be arranged in descending

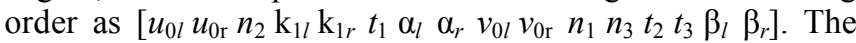
principal point $\left(u_{0 l}, v_{0 l}\right)$ and $\left(u_{0 r}, v_{0 r}\right)$, the component $n_{2}$ of the rotation vector along the ordinate axis, the first order radial distortion coefficient $\mathrm{k}_{1 l}, \mathrm{k}_{1 r}$, the component $t_{1}$ of the translation vector along the abscissa axis, and the abscissa of the 


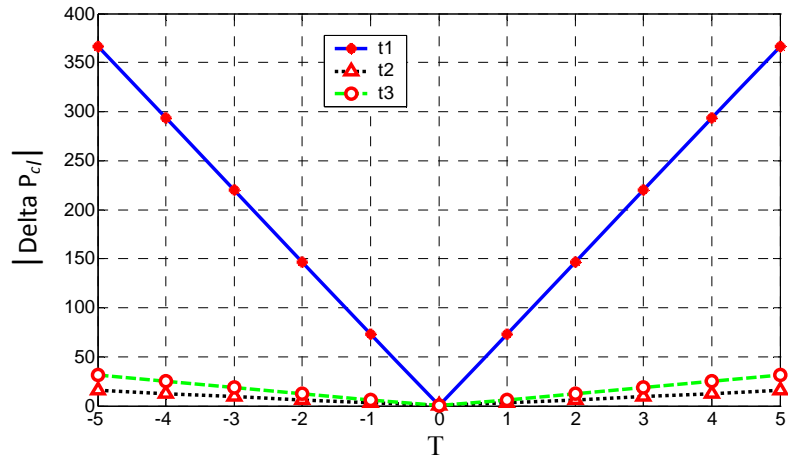

(a)

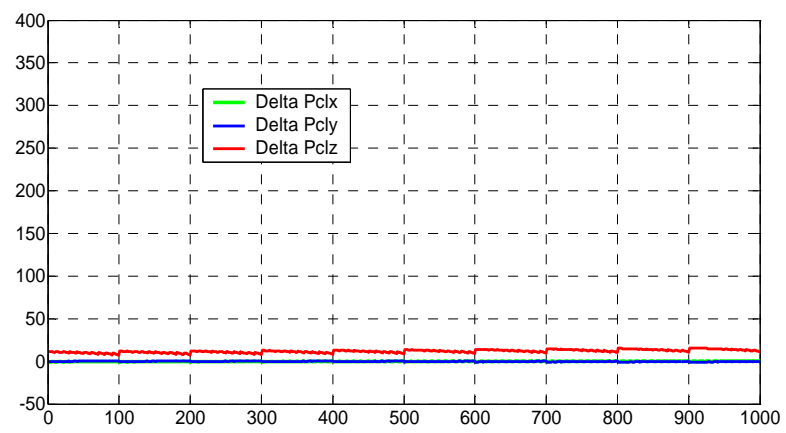

(c)

Fig. (8). Effect of translation vector

(a) Error versus deviation of the translation vector;

(b) Components of error versus point ordinal when $t 1$ changes

(c) Components of error versus point ordinal when $t 2$ changes;

(d) Components of error versus point ordinal when $t 3$ changes

principal length $\alpha_{l}, \alpha_{r}$ are the major factors affecting reconstruction accuracy. The other calibration parameters have minor effect on reconstruction accuracy.

For stereovision systems with different intrinsic or extrinsic parameters, the above simulation experiment is also conducted. The results show that the effect degree of different calibration parameters on reconstruction accuracy may change slightly. However, the major factors remain the same.

In order to satisfy accuracy requirement of a stereovision system, more attention must be paid to the major factors in the process of calibration, especially, the abscissa of the principal point $u_{0 l}, u_{0 \mathrm{r}}$, the component of the rotation vector along the ordinate axis $n_{2}$ and the component of the translation vector along the abscissa axis $t_{1}$.

\section{CONCLUSION AND DISCUSSION}

From above results of simulation experiment, the following conclusion can be drawn.

Among the calibration parameters of a typical stereovision system, the major factors affecting reconstruction accuracy include the principal point, the component of the rotation vector along the ordinate axis, the first order radial distortion coefficient, the component of the translation vector along the abscissa axis, and abscissa of the principal length.

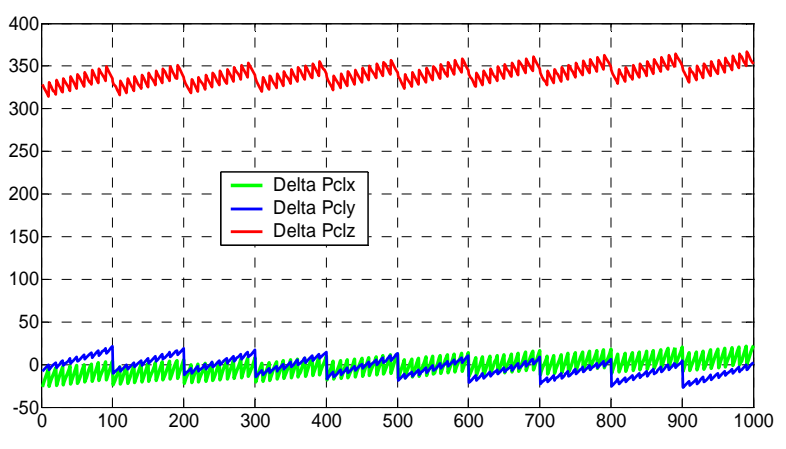

(b)

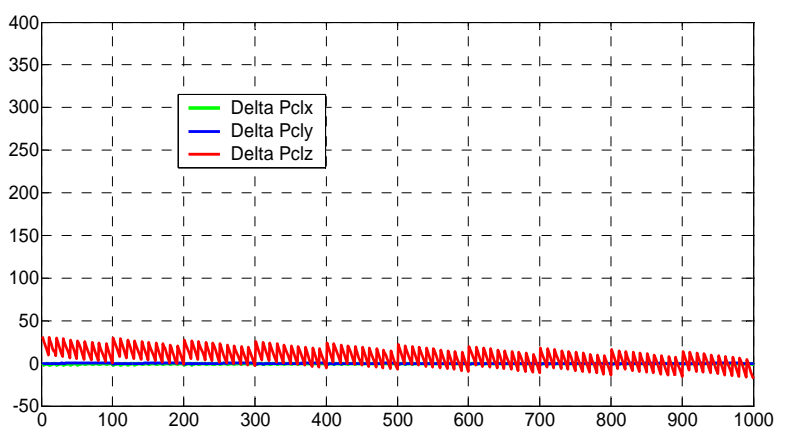

(d)

The other calibration parameters have minor effect on reconstruction accuracy.

In the process of calibration, more attention must be paid to the major factors, especially the abscissa of the principal point, the component of the rotation vector along the ordinate axis and the component of the translation vector along the abscissa axis, and their error must be controlled strictly, so that the accuracy requirement of the stereovision system can be satisfied.

\section{CONFLICT OF INTEREST}

The authors confirm that this article content has no conflicts of interest.

\section{ACKNOWLEDGMENTS}

This work is supported by Shanghai Municipal Natural Science Foundation (10ZR1413600), and Key Scientific and Technological Project of Shanghai (11dz1205600).

\section{REFERAENCES}

[1] J.I.A Yunde and Q.I.N Xiameng, "An embedded calibration stereovision system", Proc. of 2012 Intelligent Vehicles Symposium, pp. 1072-1077, 2012.

[2] W. Kim, A. Ansar, R. Steele, and R. Steinke, "Performance analysis and validation of a stereo vision system," Proc. of IEEE Int. Conf. Syst., Man, Cybern., vol. 2, pp. 1409-1416, 2005. 
[3] Giuseppe Di Leo, Consolatina Liguori, and Alfredo Paolillo, "Covariance Propagation for the Uncertainty Estimation in Stereo Vision”, IEEE Trans Instrum Meas, vol. 60, no. 5, 1664-1673, 2011.

[4] D. Scharstein and R. Szeliski, "A Taxonomy and Evaluation of Dense Two-Frame Stereo Correspondence Algorithms," Int. J. Comp Vis, vol. 47, pp.7-42, 2002.

[5] Neus Sabater, Andre's Almansa, and Jean-Michel Morel, "Meaningful Matches in Stereovision”, IEEE Trans. Pattern Anal. Mach Intell., vol. 34, no. 5, pp.930-942, 2012.
[6] J. Chen, Z. Ding, and F. Yuan, "Theoretical uncertainty evaluation of stereo reconstruction," Proc. of 2nd Int. Conf. Bioinformatics Biomed. Eng., May 16-18, pp. 2378-2381, 2008.

[7] K. Kanatani, Y. Sugaya, and H. Niitsuma, "Triangulation from two views revisited: Hartley-Sturm vs. optimal correction," In Proc. 19th Brit. Mach. Vis. Conf., Leeds, U.K., pp. 173182, 2008.

[8] R. M. Murray, Z. Li and S. S. Sastry, "A Mathematical Introduction to Robotic Manipulation”, Boca Raton, FL: CRC Press, 26-28, 1994.

Received: March 12, 2013

(C) Wang et al.; Licensee Bentham Open.

This is an open access article licensed under the terms of the Creative Commons Attribution Non-Commercial License (http://creativecommons.org/licenses/by-nc/3.0/) which permits unrestricted, non-commercial use, distribution and reproduction in any medium, provided the work is properly cited. 